\title{
On one master integral for three-loop on-shell HQET propagator diagrams with mass
}

\author{
A.G. Grozin \\ Budker Institute of Nuclear Physics, Novosibirsk, Russia, and \\ Department of Physics, University of Alberta, Edmonton, Canada \\ A.G.Grozin@inp.nsk.su
}

\section{T. Huber}

Institut für Theoretische Physik E, RWTH Aachen, D-52056 Aachen, Germany thuber@physik.rwth-aachen.de

\section{Maître}

Stanford Linear Accelerator Center, Stanford University, Stanford, CA 94309, USA maitreda@slac.stanford.edu

Abstract: An exact expression for the master integral $I_{2}$ [1] arising in three-loop onshell HQET propagator diagrams with mass is derived and its analytical expansion in the dimensional regularization parameter $\varepsilon$ is given.

Keywords: NLO Computations. 
All three-loop on-shell HQET propagator integrals with a loop of a massive quark can be reduced to a set of master integrals [1]. A reduction algorithm has been constructed by solving the integration-by-parts 2] relations using a Gröbner bases technique [3]. Some master integrals are known exactly, for the remaining ones a few terms of their respective $\varepsilon$ expansion have been calculated in Ref. [1]. In particular, for the integral

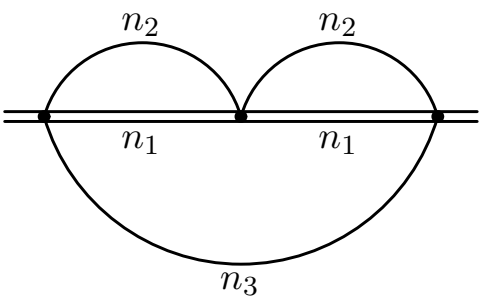

Figure 1: The integral $I_{n_{1} n_{2} n_{3}}$ $I_{2}$, the $1 / \varepsilon$ and $\mathcal{O}\left(\varepsilon^{0}\right)$ terms are known. However, in some applications more terms of its $\varepsilon$ expansion are needed (A. Czarnecki, A. Pak, work in progress). Here, we shall obtain an exact result for $I_{2}$ and additional terms of its expansion in $\varepsilon$.

We shall consider a more general integral (Fig. 1):

$$
\begin{aligned}
I_{n_{1} n_{2} n_{3}} & =\frac{1}{i \pi^{d / 2}} \int \frac{I_{n_{1} n_{2}}^{2}\left(p_{0}\right) d^{d} p}{\left(1-p^{2}-i 0\right)^{n_{3}}} \\
I_{n_{1} n_{2}}\left(p_{0}\right) & =\frac{1}{i \pi^{d / 2}} \int \frac{d^{d} k}{\left(-2\left(k_{0}+p_{0}\right)-i 0\right)^{n_{1}}\left(1-k^{2}-i 0\right)^{n_{2}}},
\end{aligned}
$$

using a method similar to the one used in $\|$ for calculating a three-loop vacuum integral. Using the HQET Feynman parametrization, we write the one-loop subdiagram as

$$
I_{n_{1} n_{2}}\left(p_{0}\right)=\frac{\Gamma\left(n_{1}+n_{2}-2+\varepsilon\right)}{\Gamma\left(n_{1}\right) \Gamma\left(n_{2}\right)} \int_{0}^{\infty} y^{n_{1}-1}\left(y^{2}-2 p_{0} y+1\right)^{2-n_{1}-n_{2}-\varepsilon} d y .
$$

Then, after the Wick rotation $p_{0}=i p_{E 0}$, we can calculate the integral in $d^{d-1} \vec{p}$ :

$$
I_{n_{1} n_{2} n_{3}}=\frac{\Gamma\left(n_{3}-3 / 2+\varepsilon\right)}{\pi^{1 / 2} \Gamma\left(n_{3}\right)} \int_{-\infty}^{+\infty} I_{n_{1} n_{2}}^{2}\left(i p_{E 0}\right)\left(1+p_{E 0}^{2}\right)^{3 / 2-n_{3}-\varepsilon} d p_{E 0} .
$$

Here $\operatorname{Re} I_{n_{1} n_{2}}^{2}\left(i p_{E 0}\right)$ is an even function of $p_{E 0} ; \operatorname{Im} I_{n_{1} n_{2}}^{2}\left(i p_{E 0}\right)$ is an odd function, and does not contribute to the integral.

The integral (2) at $p_{0}<0$ can be expressed via the hypergeometric function ${ }_{2} F_{1}$ :

$$
I_{n_{1} n_{2}}\left(p_{0}\right)=\frac{\Gamma\left(n_{1}+n_{2}-2+\varepsilon\right) \Gamma\left(n_{1}+2 n_{2}-4+2 \varepsilon\right)}{\Gamma\left(n_{2}\right) \Gamma\left(2\left(n_{1}+n_{2}-2+\varepsilon\right)\right)}{ }_{2} F_{1}\left(\begin{array}{c}
\frac{1}{2} n_{1}, \frac{1}{2} n_{1}+n_{2}-2+\varepsilon \\
n_{1}+n_{2}-\frac{3}{2}+\varepsilon
\end{array} \mid 1-p_{0}^{2}\right)
$$

(a similar expression has been derived in Ref. [5]). Its square can be expressed via ${ }_{3} F_{2}$, due to the Clausen identity. In order to perform an analytical continuation to $p_{0}=i p_{E 0}$, we re-express this ${ }_{3} F_{2}$ via three ${ }_{3} F_{2}$ functions of the inverse argument:

$$
\operatorname{Re} I_{n_{1} n_{2}}^{2}\left(i p_{E 0}\right)=R_{n_{1} n_{2}}(z), \quad z=\frac{1}{1+p_{E 0}^{2}},
$$

where

$$
R_{n_{1} n_{2}}(z)=\frac{\Gamma^{2}\left(n_{2}-2+\varepsilon\right)}{\Gamma^{2}\left(n_{2}\right)}\left(-\frac{z}{4}\right)^{n_{1}}\left[{ }_{3} F_{2}\left(\begin{array}{c}
n_{1}, \frac{5}{2}-n_{2}-\varepsilon, 5-n_{1}-2 n_{2}-2 \varepsilon \\
3-n_{2}-\varepsilon, 5-2 n_{2}-2 \varepsilon
\end{array} \mid z\right)\right.
$$




$$
\begin{aligned}
& -2 A_{n_{1} n_{2}}(z) \cos (\pi \varepsilon){ }_{3} F_{2}\left(\begin{array}{c}
\frac{1}{2}, 3-n_{1}-n_{2}-\varepsilon, n_{1}+n_{2}-2+\varepsilon \\
3-n_{2}-\varepsilon, n_{2}-1+\varepsilon
\end{array} \mid z\right) \\
& \left.+A_{n_{1} n_{2}}^{2}(z) \cos (2 \pi \varepsilon){ }_{3} F_{2}\left(\begin{array}{c}
1-n_{1}, n_{2}-\frac{3}{2}+\varepsilon, n_{1}+2 n_{2}-4+2 \varepsilon \\
n_{2}-1+\varepsilon, 2 n_{2}-3+2 \varepsilon
\end{array} \mid z\right)\right],
\end{aligned}
$$

and

$$
A_{n_{1} n_{2}}(z)=\frac{\pi}{\sin (\pi \varepsilon)} \frac{\Gamma\left(n_{1}+2 n_{2}-4+2 \varepsilon\right)}{\Gamma\left(n_{1}\right) h\left(n_{2}-2+\varepsilon\right)}\left(\frac{z}{4}\right)^{n_{2}-2+\varepsilon}
$$

with $h(x)=x \Gamma^{2}(x)$. This result simplifies at $n_{1}=1$ :

$$
\begin{aligned}
& R_{1 n_{2}}(z)=-\frac{\Gamma^{2}\left(n_{2}-2+\varepsilon\right)}{4 \Gamma^{2}\left(n_{2}\right)} z\left[{ }_{3} F_{2}\left(\begin{array}{c}
1, \frac{5}{2}-n_{2}-\varepsilon, 4-2 n_{2}-2 \varepsilon \\
3-n_{2}-\varepsilon, 5-2 n_{2}-2 \varepsilon
\end{array} \mid z\right)\right. \\
& \left.-2 A_{1 n_{2}}(z) \cos (\pi \varepsilon){ }_{2} F_{1}\left(\begin{array}{c}
\frac{1}{2}, 2-n_{2}-\varepsilon \\
3-n_{2}-\varepsilon
\end{array} \mid z\right)+A_{1 n_{2}}^{2}(z) \cos (2 \pi \varepsilon)\right] \text {. }
\end{aligned}
$$

Calculating the integral

$$
I_{n_{1} n_{2} n_{3}}=\frac{\Gamma\left(n_{3}-3 / 2+\varepsilon\right)}{\pi^{1 / 2} \Gamma\left(n_{3}\right)} \int_{0}^{1} R_{n_{1} n_{2}}(z) z^{n_{3}-3+\varepsilon}(1-z)^{-1 / 2} d z
$$

term-by-term, we obtain

$$
\begin{aligned}
& I_{n_{1} n_{2} n_{3}}=\frac{(-1)^{n_{1}}}{\Gamma^{2}\left(n_{2}\right) \Gamma\left(n_{3}\right)}\left[\frac{h\left(n_{1}+n_{3}-2+\varepsilon\right) \Gamma^{2}\left(n_{2}-2+\varepsilon\right) \Gamma\left(2 n_{3}-3+2 \varepsilon\right)}{\Gamma\left(n_{3}-1+\varepsilon\right) \Gamma\left(2 n_{1}+2 n_{3}-3+2 \varepsilon\right)}\right. \\
& \times{ }_{4} F_{3}\left(\begin{array}{c}
n_{1}, \frac{5}{2}-n_{2}-\varepsilon, n_{1}+n_{3}-2+\varepsilon, 5-n_{1}-2 n_{2}-2 \varepsilon \mid \\
n_{1}+n_{3}-\frac{3}{2}+\varepsilon, 5-2 n_{2}-2 \varepsilon, 3-n_{2}-\varepsilon
\end{array} \mid \begin{array}{c}
1
\end{array}\right) \\
& -2 \pi \frac{\cos (\pi \varepsilon)}{\sin (\pi \varepsilon)} \frac{h\left(n_{1}+n_{2}+n_{3}-4+2 \varepsilon\right) \Gamma\left(n_{1}+2 n_{2}-4+2 \varepsilon\right) \Gamma\left(2 n_{3}-3+2 \varepsilon\right)}{\left(n_{2}-2+\varepsilon\right) \Gamma\left(n_{1}\right) \Gamma\left(n_{3}-1+\varepsilon\right) \Gamma\left(2 n_{1}+2 n_{2}+2 n_{3}-7+4 \varepsilon\right)} \\
& \times{ }_{4} F_{3}\left(\begin{array}{c}
\frac{1}{2}, n_{1}+n_{2}+n_{3}-4+2 \varepsilon, 3-n_{1}-n_{2}-\varepsilon, n_{1}+n_{2}-2+\varepsilon \\
n_{1}+n_{2}+n_{3}-\frac{7}{2}+2 \varepsilon, 3-n_{2}-\varepsilon, n_{2}-1+\varepsilon
\end{array}\right) \\
& +\pi^{2} \frac{\cos (2 \pi \varepsilon)}{\sin ^{2}(\pi \varepsilon)} \frac{h\left(n_{1}+2 n_{2}+n_{3}-6+3 \varepsilon\right) \Gamma^{2}\left(n_{1}+2 n_{2}-4+2 \varepsilon\right) \Gamma\left(2 n_{3}-3+2 \varepsilon\right)}{\Gamma^{2}\left(n_{1}\right) \Gamma^{2}\left(n_{2}-1+\varepsilon\right) \Gamma\left(n_{3}-1+\varepsilon\right) \Gamma\left(2 n_{1}+4 n_{2}+2 n_{3}-11+6 \varepsilon\right)} \\
& \left.\times{ }_{4} F_{3}\left(\begin{array}{c}
1-n_{1}, n_{2}-\frac{3}{2}+\varepsilon, n_{1}+2 n_{2}-4+2 \varepsilon, n_{1}+2 n_{2}+n_{3}-6+3 \varepsilon \\
n_{2}-1+\varepsilon, 2 n_{2}-3+2 \varepsilon, n_{1}+2 n_{2}+n_{3}-\frac{11}{2}+3 \varepsilon
\end{array}\right)\right] .
\end{aligned}
$$

In particular,

$$
\begin{aligned}
& I_{1 n_{2} n_{3}}=-\frac{1}{\Gamma^{2}\left(n_{2}\right) \Gamma\left(n_{3}\right)}\left[\frac{\Gamma^{2}\left(n_{2}-2+\varepsilon\right) \Gamma\left(n_{3}-1+\varepsilon\right)}{2\left(2 n_{3}-3+2 \varepsilon\right)}\right. \\
& \times{ }_{4} F_{3}\left(\begin{array}{c|c}
1, \frac{5}{2}-n_{2}-\varepsilon, n_{3}-1+\varepsilon, 4-2 n_{2}-2 \varepsilon \\
n_{3}-\frac{1}{2}+\varepsilon, 5-2 n_{2}-2 \varepsilon, 3-n_{2}-\varepsilon
\end{array}\right) \\
& -2 \pi \frac{\cos (\pi \varepsilon)}{\sin (\pi \varepsilon)} \frac{h\left(n_{2}+n_{3}-3+2 \varepsilon\right) \Gamma\left(2 n_{2}-3+2 \varepsilon\right) \Gamma\left(2 n_{3}-3+2 \varepsilon\right)}{\left(n_{2}-2+\varepsilon\right) \Gamma\left(n_{3}-1+\varepsilon\right) \Gamma\left(2 n_{2}+2 n_{3}-5+4 \varepsilon\right)}
\end{aligned}
$$




$$
\begin{gathered}
\times{ }_{3} F_{2}\left(\begin{array}{c}
\frac{1}{2}, n_{2}+n_{3}-3+2 \varepsilon, 2-n_{2}-\varepsilon \\
n_{2}+n_{3}-\frac{5}{2}+2 \varepsilon, 3-n_{2}-\varepsilon
\end{array} \mid 1\right) \\
\left.+\pi^{2} \frac{\cos (2 \pi \varepsilon)}{\sin ^{2}(\pi \varepsilon)} \frac{h\left(2 n_{2}+n_{3}-5+3 \varepsilon\right) \Gamma^{2}\left(2 n_{2}-3+2 \varepsilon\right) \Gamma\left(2 n_{3}-3+2 \varepsilon\right)}{\Gamma^{2}\left(n_{2}-1+\varepsilon\right) \Gamma\left(n_{3}-1+\varepsilon\right) \Gamma\left(4 n_{2}+2 n_{3}-9+6 \varepsilon\right)}\right] .
\end{gathered}
$$

For example, let us consider the convergent integral $I_{122}$. Using the reduction procedure of Ref. [1], we can relate it to the master integral $I_{2} \equiv I_{111}$ :

$$
I_{122}=-\frac{(d-3)^{2}(d-4)(3 d-8)(3 d-10)}{8(3 d-11)(3 d-13)} I_{2}
$$

From Eq. (11) we have

$$
\begin{aligned}
\frac{I_{122}}{\Gamma^{3}(1+\varepsilon)}= & -\frac{1}{2 \varepsilon^{2}}\left[\frac{1}{1+2 \varepsilon}{ }_{4} F_{3}\left(\begin{array}{c}
1, \frac{1}{2}-\varepsilon, 1+\varepsilon,-2 \varepsilon \\
\frac{3}{2}+\varepsilon, 1-\varepsilon, 1-2 \varepsilon
\end{array} \mid 1\right)\right. \\
& -\frac{2}{1+4 \varepsilon} \frac{\Gamma^{2}(1-\varepsilon) \Gamma^{3}(1+2 \varepsilon)}{\Gamma^{2}(1+\varepsilon) \Gamma(1-2 \varepsilon) \Gamma(1+4 \varepsilon)}{ }_{3} F_{2}\left(\begin{array}{c}
\frac{1}{2}, 1+2 \varepsilon,-\varepsilon \\
\frac{3}{2}+2 \varepsilon, 1-\varepsilon
\end{array} \mid 1\right) \\
& \left.+\frac{1}{1+6 \varepsilon} \frac{\Gamma^{2}(1-\varepsilon) \Gamma^{4}(1+2 \varepsilon) \Gamma(1-2 \varepsilon) \Gamma^{2}(1+3 \varepsilon)}{\Gamma^{4}(1+\varepsilon) \Gamma(1+4 \varepsilon) \Gamma(1-4 \varepsilon) \Gamma(1+6 \varepsilon)}\right] .
\end{aligned}
$$

There are several methods to expand the hypergeometric functions in $\varepsilon[6,7,8]$. We first follow the method of Ref. [7]. First we express $\Gamma$ functions in the hypergeometric series as exponents containing $S$-sums

$$
S\left(N ; k_{1}, \ldots, k_{l} ; x_{1}, \ldots, x_{l}\right)=\sum_{N \geqslant n_{1} \geqslant \cdots \geqslant n_{l} \geqslant 1} \frac{x_{1}^{n_{1}}}{n_{1}^{k_{1}}} \cdots \frac{x_{l}^{n_{l}}}{n_{l}^{k_{l}}} .
$$

Some indices are integer at $\varepsilon \rightarrow 0$ and some are half-integer; therefore, we get both $S(n ; k ; 1)$ and $S(2 n ; k ; 1)$. We can re-express $S(n ; k ; 1)$ via $S(2 n ; k ; \pm 1)$ by inserting $\left(1+(-1)^{n_{1}}\right) / 2$ under the summation sign:

$$
\begin{aligned}
& { }_{3} F_{2}\left(\begin{array}{c}
\frac{1}{2}, 1+2 \varepsilon,-\varepsilon \\
\frac{3}{2}+2 \varepsilon, 1-\varepsilon
\end{array} \mid \begin{array}{l}
1 \\
2
\end{array}\right)=1 \\
& -\varepsilon(1+4 \varepsilon) \sum_{n=1}^{\infty} \frac{1}{(n-\varepsilon)(2 n+1+4 \varepsilon)} \exp \left[-\sum_{k=1}^{\infty} \frac{(-4 \varepsilon)^{k}}{k} S(2 n ; k ;-1)\right] \\
& { }_{4} F_{3}\left(\begin{array}{c}
1, \frac{1}{2}-\varepsilon, 1+\varepsilon,-2 \varepsilon \\
\frac{3}{2}+\varepsilon, 1-\varepsilon, 1-2 \varepsilon
\end{array} \mid 1\right)=1 \\
& -2 \varepsilon(1+2 \varepsilon) \sum_{n=1}^{\infty} \frac{1}{(n-2 \varepsilon)(2 n+1+2 \varepsilon)} \exp \left[\sum_{k=1}^{\infty} \frac{(2 \varepsilon)^{k}}{k}\left(1-(-1)^{k}\right) S(2 n ; k ;-1)\right]
\end{aligned}
$$

Products of $S$-sums with upper limit $2 n$ are expressed in terms of single $S$-sums by means of the well-known algebra [9, 10, 11]. We expand the rational factors in Eq. (15) in $\varepsilon$, and then expand them into partial fractions. After that, separate sums may diverge, and we 
introduce an upper limit $N$ instead of $\infty$ in the outermost sum. Sums with $1 / n^{k}$ can be re-written as sums in $n$ to $2 N$ by inserting $\left(1+(-1)^{n}\right) / 2$, and those with $1 /(2 n+1)^{k}$ by inserting $\left(1-(-1)^{n}\right) / 2$ :

$$
\begin{aligned}
& \sum_{n=1}^{N} \frac{1}{n^{k}} S\left(2 n ; k_{1}, \ldots, k_{l} ; x_{1}, \ldots, x_{l}\right) \\
& =2^{k-1}\left[S\left(2 N ; k, k_{1}, \ldots, k_{l} ; 1, x_{1}, \ldots, x_{l}\right)+S\left(2 N ; k, k_{1}, \ldots, k_{l} ;-1, x_{1}, \ldots, x_{l}\right)\right], \\
& \sum_{n=1}^{N} \frac{1}{(2 n+1)^{k}} S\left(2 n ; k_{1}, \ldots, k_{l} ; x_{1}, \ldots, x_{l}\right) \\
& =\frac{1}{2}\left[S\left(2 N ; k, k_{1}, \ldots, k_{l} ; 1, x_{1}, \ldots, x_{l}\right)-S\left(2 N ; k, k_{1}, \ldots, k_{l} ;-1, x_{1}, \ldots, x_{l}\right)\right. \\
& \left.\quad-S\left(2 N ; k+k_{1}, k_{2}, \ldots, k_{l} ; x_{1}, x_{2}, \ldots, x_{l}\right)+S\left(2 N ; k+k_{1}, k_{2}, \ldots, k_{l} ;-x_{1}, x_{2}, \ldots, x_{l}\right)\right]
\end{aligned}
$$

(terms vanishing at $N \rightarrow \infty$ are omitted here). After that, all sums divergent at $N \rightarrow \infty$ cancel; in the remaining sums, we may set $N=\infty$. They are related to the Euler-Zagier sums [9, 10].

Yet another method of expanding certain classes of hypergeometric functions about half-integer parameters is provided by the algorithm of Ref. [8], by means of which we have calculated the expansion of Eq. (13) up to order $\mathcal{O}\left(\varepsilon^{7}\right)$. After factoring out an appropriate combination of pre-factors, the expansion reads

$$
\begin{aligned}
\frac{I_{122}}{\Gamma^{3}(1+\varepsilon)}= & \frac{\pi^{2}}{3(1+6 \varepsilon)} \\
& \times\left[1-\pi^{2} \varepsilon^{2}+48 \zeta_{3} \varepsilon^{3}-\frac{38 \pi^{4}}{15} \varepsilon^{4}-48\left(\pi^{2} \zeta_{3}-30 \zeta_{5}\right) \varepsilon^{5}+\left(1152 \zeta_{3}^{2}-\frac{4793 \pi^{6}}{945}\right) \varepsilon^{6}\right. \\
& \left.+\left(39312 \zeta_{7}-\frac{608 \pi^{4} \zeta_{3}}{5}-1440 \pi^{2} \zeta_{5}\right) \varepsilon^{7}+\mathcal{O}\left(\varepsilon^{8}\right)\right]
\end{aligned}
$$

In order to check the correctness of this expansion, we have converted the hypergeometric functions in Eq. (13) to single Mellin-Barnes representations and subsequently obtained the coefficients numerically [12]. We find agreement to at least 14 decimal digits.

It is now straightforward to obtain the expansion of the master integral $I_{2}$ from Eq. (12):

$$
\frac{I_{2}}{\Gamma^{3}(1+\varepsilon)}=-\frac{\pi^{2}}{6}\left[\frac{1}{\varepsilon}+\frac{5}{2}-\left(\pi^{2}+\frac{21}{4}\right) \varepsilon+\left(48 \zeta_{3}-\frac{5}{2} \pi^{2}-\frac{599}{8}\right) \varepsilon^{2}+\cdots\right] .
$$

The first two terms have been obtained in Ref. [1] by a completely different method. The next one has been found by A. Pak from the requirement of cancellation of $1 / \varepsilon$ poles in a physical calculation. The last term is then required to calculate the corresponding finite part. Yet higher terms in the expansion of $I_{2}$ can be obtained from Eq. (16).

As a closing remark we would like to mention that the expansion in Eq. (16) is found to agree up to order $\mathcal{O}\left(\varepsilon^{7}\right)$ with the expansion of the simple formula

$$
\frac{I_{122}}{\Gamma^{3}(1+\varepsilon)}=\frac{\pi^{2}}{3} \frac{\Gamma^{3}(1+2 \varepsilon) \Gamma^{2}(1+3 \varepsilon)}{\Gamma^{6}(1+\varepsilon) \Gamma(2+6 \varepsilon)} .
$$


We do not know any analytical proof of this result, but high-precision numerical tests of Eqs. (13) and (18) for various values of $\varepsilon$ on the real axis and in the complex plane strengthen the conjecture that this result might indeed be valid to all orders. However, until an analytical proof of Eq. (18) is found, we are guaranteed to obtain the correct expansion only by the analytically derived expression (13).

\section{Acknowledgments}

A.G. is grateful to O.V. Tarasov for the suggestion to use a method similar to Ref. [A]; to A. Pak for communicating his result for the $\mathcal{O}(\varepsilon)$ term in Eq. (17) and for motivation to calculate the $\mathcal{O}\left(\varepsilon^{2}\right)$ term; to A.I. Davydychev for an advice on hypergeometric functions; and to V.A. Smirnov for an independent check of some calculations. T.H. is supported by Deutsche Forschungsgemeinschaft, SFB/TR 9 "Computergestützte Theoretische Teilchenphysik". D.M. is supported by the SNF under contract PBZH2-117028 and by the US Departement of Energy under contract DE-AC02-76SF00515.

\section{References}

[1] A.G. Grozin, A.V. Smirnov, V.A. Smirnov, Decoupling of heavy quarks in HQET, J. High Energy Phys. 11 (2006) 022 hep-ph/0609280.

[2] F.V. Tkachov, A theorem on analytical calculability of four loop renormalization group functions, Phys. Lett. B 100 (1981) 65:

K.G. Chetyrkin, F.V. Tkachov, Integration by parts: The algorithm to calculate $\beta$-functions in 4 loops, Nucl. Phys. B 192 (1981) 159.

[3] A.V. Smirnov and V.A. Smirnov, Applying Gröbner bases to solve reduction problems for Feynman integrals, J. High Energy Phys. 01 (2006) 001 hep-lat/0509187;

A.V. Smirnov, An algorithm to construct Gröbner bases for solving integration by parts relations, J. High Energy Phys. 04 (2006) 026 hep-ph/0602078.

[4] D.J. Broadhurst, Three-loop on-shell charge renormalization without integration: $\Lambda_{Q E D}^{\overline{M S}}$ to four loops, Z. Physik C 54 (1992) 599.

[5] J. Zupan, One loop scalar functions in the heavy quark effective theory, Eur. Phys. J. C 25 (2002) 233 hep-ph/0202135.

[6] A.I. Davydychev, M.Yu. Kalmykov, Massive Feynman diagrams and inverse binomial sums, Nucl. Phys. B 699 (2004) 3 hep-th/0303162;

M.Yu. Kalmykov, Gauss hypergeometric function: Reduction, epsilon-expansion for integer/half-integer parameters and Feynman diagrams, J. High Energy Phys. 04 (2006) 056 hep-th/0602028;

M.Yu. Kalmykov, B.F.L. Ward, S. Yost, All order epsilon-expansion of Gauss hypergeometric functions with integer and half/integer values of parameters, J. High Energy Phys. 02 (2007) 040 hep-th/0612240.

[7] S. Weinzierl, Expansion around half integer values, binomial sums and inverse binomial sums, J. Math. Phys. 45 (2004) 2656 hep-ph/0402131.

[8] T. Huber, D. Maître, work in preparation. 
[9] J.M. Borwein, D.M. Bradley, D.J. Broadhurst, Evaluation of $k$-fold Euler/Zagier sums: A compendium of results for arbitrary $k$, Electronic J. Combinatorics 4(2) (1997) \#R5 hep-th/9611004;

D.J. Broadhurst, Conjectured enumeration of irreducible multiple zeta values, from knots and Feynman diagrams, hep-th/9612012;

J.M. Borwein, D.M. Bradley, D.J. Broadhurst, P. Lisoněk, Special values of multiple polylogarithms, Trans. Am. Math. Soc. 353 (2001) 907 math.CA/9910045.

[10] J.A.M. Vermaseren, Harmonic sums, Mellin transforms and integrals, Int. J. Mod. Phys. A 14 (1999) 2037 hep-ph/9806280.

[11] S. Moch, P. Uwer, S. Weinzierl, Nested sums, expansion of transcendental functions and multiscale multiloop integrals, J. Math. Phys. 43 (2002) 3363 hep-ph/0110083.

[12] M. Czakon, Automatized analytic continuation of Mellin-Barnes integrals, Comput. Phys. Commun. 175 (2006) 559 hep-ph/0511200. 